Original Contribution

\title{
SPONTANEOUS TRANSVAGINAL SIGMOID COLON HERNIATION: A RARE CASE OF SURGICAL EMERGENCY
}

\author{
Iv. Novakov* \\ Department of Special Surgery, Medical University - Plovdiv, Bulgaria
}

\begin{abstract}
The evisceration of abdominal organs through the vagina is a rare complication of hysterectomy. The aim of this publication is to report an exceedingly rare event of spontaneous transvaginal sigmoid colon herniation in postmenopausal women. A 74-year-old woman after hysterectomy twenty-seven years ago and spontaneous transvaginal sigmoid colon herniation is presented. What makes this case original and interesting is the type of herniated abdominal organ, lack of precipitating factor and very long period after abdominal hysterectomy. The presented case also demonstrates the options of laparotomy as an operative approach in the management of vaginal herniation.
\end{abstract}

Key words: abdominal hysterectomy, vaginal evisceration, redundant sigmoid colon, laparotomy.

\section{INTRODUCTION}

The evisceration of abdominal organs through the vagina is a rare complication of hysterectomy. When it does occur, it requires urgent surgical intervention. The most common abdominal organ to prolapse through the vagina is the small bowel followed by the omentum, colon, fallopian tubes, and appendix (1-4). The aim of this publication is to report an exceedingly rare event of spontaneous transvaginal sigmoid colon herniation in postmenopausal woman.

\section{CASE PRESENTATION}

A 74-year-old woman with a sudden abdominopelvic pain was examined in the emergency department of University hospital. The patient's detailed history revealed that abdominal pain was appeared at the time of regular household activities and immediately after the pain a sensation of "a protruding mass between the legs" was felt. The woman noticed a loop of bowel protruding from her vagina and she was brought to the emergency department. Neither constipation nor coughing episode that would increase the intra-abdominal pressure and no history of abdominal or vaginal trauma were observed. The woman had undergone a total

\footnotetext{
*Correspondence to: Department of Special Surgery, Medical University - Plovdiv, Bulgaria, e-mail: inovakov2003@yahoo.com, mobile phone: $+359887575487$
}

abdominal hysterectomy for a benign pathology twenty seven years ago.

On examination the woman's abdomen was distended and tender in the supra-pubic area. Perineal exam revealed sigmoid colon prolapsing through her vagina. The prolapsed sigmoid colon appeared with signs of impaired venous return - edematous, congested and ecchymotic (Figure 1).

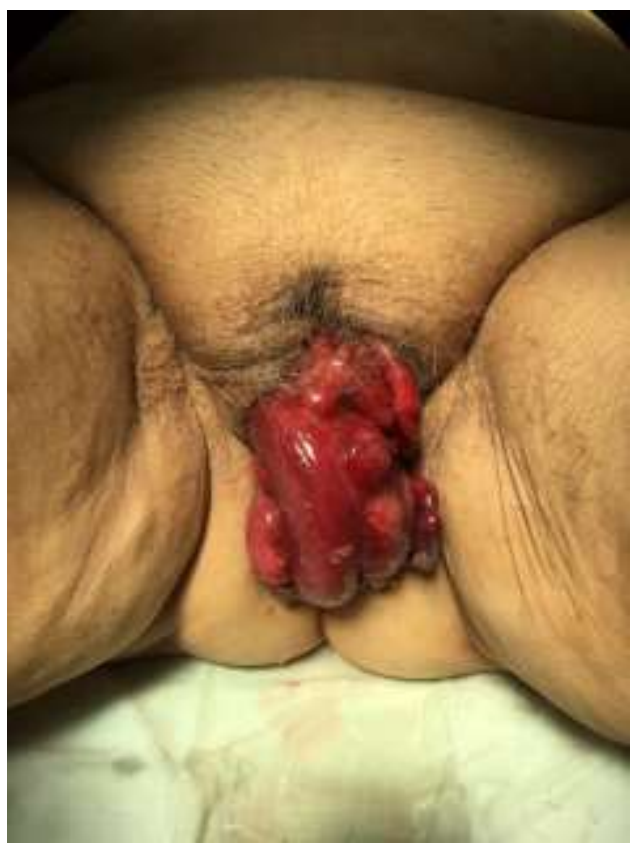

Figure 1. Physical examination revealing edematous, congested and ecchymotic sigmoid colon herniated through the vagina. 
The vital signs of the woman were stable (at admission in the emergency department her blood pressure was $110 \times 70 \mathrm{mmHg}$ and the heart rate was $88 \mathrm{bpm}$ ).

The prolapsed colon was wrapped with warm, sterile and saline soaked gauze and the woman was transferred to the operating room of the Department of Special Surgery.

An examination under general anesthesia revealed that reduction of the herniated sigmoid colon via the vaginal defect was impossible. A laparotomy was performed through a midline abdominal incision. The herniated sigmoid colon was reduced into the abdomen with difficulty (with a help of an assistant who pushed back the herniated colon transvaginally). The peritoneal exploration, during the exploration it was observed that the sigmoid colon was edematous, thick-walled but with no evidence of necrosis (Figure 2). When assessed, the length of the sigmoid colon was approximately estimated to be more than $70 \mathrm{~cm}$.

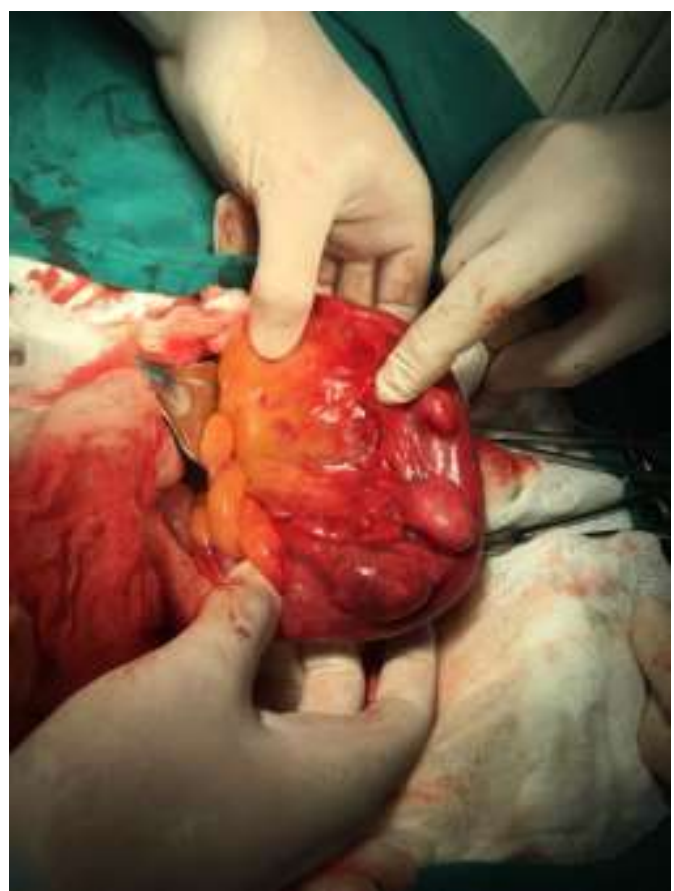

Figure 2. Operative finding: edematous, thickwalled, but viable sigmoid colon after its reposition.

A complete vaginal cuff dehiscence (separation of the entire length of the vaginal cuff) was established. The vaginal cuff edges were sharply cut until fresh tissue was visualized and the vaginal vault defect was closed with non-absorbable interrupted sutures (placed at least $1 \mathrm{~cm}$ from the cuff edge in fullthickness bites). A French 26 tube drain was placed into the Douglas pouch.

Urinary catheter was inserted after closing the abdomen. Broad-spectrum antibiotics were postoperatively given for five days. The woman had no postoperative complications and was discharged from the hospital after eight days.

\section{DISCUSSION}

The evisceration of abdominal contents through the vagina, presented in this case, is a rare, life-threatening surgical complication of hysterectomy. The etiology of transvaginal herniation still remains unclear due to limited reports in the literature. This pathology is described as in the pre-menopausal as well as in the post-menopausal women $(5,6)$.

In pre-menopausal women, the transvaginal evisceration after hysterectomy is frequently associated with sexual activity. The main reported cause is trauma of the vagina due to rough coitus (3).

The risk of transvaginal evisceration is increased in postmenopausal women (more than $70 \%$ of reported cases) / $(6,7)$. Weakening of vaginal tissue, caused by genital atrophies, is the main predisposing factor for this complication in post-menopausal women. Postmenopausal vagina is thin, scarred, foreshortened, and has diminished vascularity, and it is more prone to rupture. An increase in intra-abdominal pressure, induced by coughing, defecating, or falling is the precipitating factor for transvaginal herniation in postmenopausal women. Quite rare, the vaginal evisceration in post-menopausal women can occur spontaneously (without an increase of intra-abdominal pressure), as in the present case.

According to the literature review, the time of vaginal herniation in postmenopausal women varies in rage of 3 days to 360 months after abdominal hysterectomy (1-5). Interestingly, in the presented case vaginal evisceration is expressed twenty seven years after hysterectomy which is a relatively long period.

The most common abdominal organ to prolapse through the vagina is the small bowel (particularly the ileum). Other organs, underwent to trasvaginal herniation, such as the omentum, fallopian tubes, epiploic appendices, appendix and colon are also described (8-12). Sigmoid colon is an 
exceedingly rare reported viscus, prolapsed through the vagina. For the purpose of this publication, a computer-based search of the English literature was performed. Only three published cases of transvaginal sigmoid colon evisceration were found (1-3). Such rare manifestation increased the value of the presented case.

It is established that the sigmoid colon is elongated in elderly. Along with this, distance between junction points of sigmoid mesocolon shortened. These predispose greater mobility of the sigmoid colon. Namely, the redundant sigmoid colon, observed in the presented case was considered as the factor that caused herniation of the colon through the vagina of that 74 year-old woman.

Vaginal evisceration is a surgical emergency, and immediate recognition and surgical repair are crucial for its successful management. The appropriate management of evisceration includes a thorough assessment of the herniated organ with its reducing back into the abdomen and surgical repair of the vaginal defect (3-5). In cases of disability of the surgeon to reduce prolapsed bowel vaginally, the abdominal approach is applicable. Midline vertical incision, chosen in the presented case, ensures access to the vaginal defect with its closing, reducing the bowel into the abdomen, complete inspection of the entire abdomen with its copious lavage.

In the management of vaginal herniation, the surgeon's attention should be focused on closing the vaginal defect. The vaginal edges should be excised leaving fresh, viable tissue $(13,14)$. In the presented case, interrupted sutures with monofilament non-absorbable material closed the vaginal cuff. This manner of closing the vaginal defect is less likely to cut through unhealthy edematous tissues. Finally, the use of pelvic drain is essential to prevent potential Douglas abscess formation. It was performed in this case.

\section{CONCLUSION}

In conclusion, this publication presents one rare complication of hysterectomy in postmenopausal women. What makes this case original and interesting? At first, it is a case of extremely rare herniated abdominal organ through the vagina sigmoid colon. On the second, it is a case of spontaneous vaginal herniation - without precipitating factor and finally with a very long period after abdominal hysterectomy. The presented case also demonstrates the options of laparotomy as an operative approach in the management of vaginal herniation.

\section{REFERENCES}

1. Hur HC, Lightfoot M, McMillin MG. Vaginal cuff dehiscence evisceration: a review of the literature. Curr Opin Obstet Gynecol, 28:297303, 2016.

2. Ramirez PT, Klemer DP. Vaginal evisceration after hysterectomy: A literature review. Obstet Gynecol Surv, 57:462-467, 2002.

3. Uccella S, Ceccaroni M, Cromi A, et al. Vaginal cuff dehiscence in a series of 12,398 hysterectomies: effect of different types of colpotomy and vaginal closure. Obstet Gynecol, 120:516-523, 2012.

4. CeccaroniM, Berretta R, Malzoni M, et al. Vaginal cuff dehiscence after hysterectomy: a multicenter retrospective study. Eur J Obstetr Gynecol, 158:308-313, 2011.

5. O'Brien LM, Bellin LS, Isenberg GA, Goldstein SD: Spontaneous transvaginal small-bowel evisceration after perineal proctectomy: Report of a case and review of the literature. Dis Colon Rectum, 45:698-699, 2002.

6. Kowalski LD, Seski JC, Timmins PF et al. Vaginal evisceration: presentation and management in postmenopausal women. $\mathrm{J} \mathrm{Am}$ Coll Surg, 183:225-229, 1996.

7. Ho PW, Lee CW. Transvaginal small bowel evisceration and strangulation. ANZ J Surg, 78:726-727, 2008.

8. Dawlatly B, Lavie O, Lopes A. Transvaginal evisceration of small bowel after radical hysterectomy and pelvic lymphadenectomy. Gynecol Oncol, 73:165-166, 1999.

9. Woo Dae Kang, Seok Mo Kim, Ho Sun Choi. Vaginal evisceration after radical hysterectomy and adjuvant radiation. $J$ Gynecol Oncol, 20:63-64, 2009.

10.10. Yan Zhou, Yichao Zhang, Weicheng Liu. Spontaneous vaginal cuff dehiscence and evisceration of multiple organs - a case report. Medicine, 97:50-55, 2018.

11. Rajesh S, Kalu E, Jin B, et al. Evisceration 5 years post abdominal hysterectomy. J Obstetr Gynaecol Res, 34:425-427, 2008.

12. Ben Safta Y, Ghalleb M, Baccari A et al. Vaginal cuff dehiscence and evisceration 11 years after a radical hysterectomy: A case report. Int J Surg Case Rep, 41:234-237, 2017.

13. Matthews CA, Kenton K. Treatment of vaginal cuff evisceration. Obstet Gynecol, 124:705-708, 2014.

14. Ferrara PC, Thibodeau LG. Vaginal evisceration. J Emerg Med, 17:665-667, 1999. 
NOVAKOVIV. 